\title{
Use of a Quartz Crystal Microbalance for Studying the Effects of Propyl Gallate on the Interactions of Interleukin-6 and Its Soluble Receptor
}

\section{Su Liu' ${ }^{1,2}$, Jiadong Huang ${ }^{2,3}$, Qiang $\mathrm{Chen}^{4}$ and Jinghua $\mathrm{Yu}^{2 *}$}

${ }^{1}$ School of Resources and Environment, University of Jinan, Jinan 250022, P. R. China

${ }^{2}$ Key Laboratory of Chemical Sensing \& Analysis in Universities of Shandong, School of Chemistry and Chemical Engineering, University of Jinan, Jinan 250022, P. R. China

${ }^{3}$ School of Biological Science and Technology, University of Jinan, Jinan 250022, P. R. China

${ }^{4}$ Laboratory of Biosensors, College of Life Sciences, Nankai University, Tianjin 300071, P. R. China

\begin{abstract}
Quartz Crystal Microbalance (QCM) is known as a surface sensitive analytical device capable of on-line monitoring of interfacial reactions. To study the interaction between interleukin-6 (IL-6) and its soluble receptor (sIL-6R), as well as the effects of propyl gallate on IL-6/sIL-6R binding based on it, a QCM was employed to monitor the binding responses. In the experiments, sIL-6R was immobilized on the surface of QCM substrate, then different concentrations of IL-6 were added onto the substrate, respectively. The response frequency shift caused by the binding between IL- 6 and sIL-6R was recorded. The results showed that SIL-6R was immobilized on the substrate surface with the density of $3.43 \mathrm{ng} / \mathrm{mm}^{2}$, IL-6 then bound to the immobilized sIL-6R specifically. FAST fit program was used to analyse the data obtained of the association of IL- 6 and immobilized sIL-6R, fitting it to the biphasic kinetic equations. Kinetic constants for IL-6 binding to sIL-6R were determined from the plots of $\mathrm{K}_{\text {on }}$ versus the concentration of IL-6, $\mathrm{k}_{\text {ass }}=3.41 \times 10^{4} \mathrm{M}^{-1} \mathrm{~s}^{-1}$, $\mathrm{K}_{\text {diss }}=1.80 \times 10^{-2} \mathrm{~s}^{-1}$, thus, $\mathrm{K}_{\mathrm{D}}=5.28 \times 10^{-7} \mathrm{M}, \mathrm{K}_{\mathrm{A}}=1.89 \times 10^{6} \mathrm{M}^{-1}$. Furthermore, the effects of propyl gallate $(\mathrm{PG})$ on interaction of IL-6 with sIL-6R were studied. The results showed that PG was able to enhance the binding between IL-6 and sIL$6 \mathrm{R}$. We propose that the interaction between IL-6 and SIL-6R is one of the targets which PG can act on in vivo. The QCM offers an effective way and a new clue to the study on the mechanism of propyl gallate at molecular level.
\end{abstract}

Keywords: Interleukin-6 (IL-6); Soluble interleukin-6 receptor (sIL6R); Propyl gallate; QCM; Interaction; Kinetic analysis

\section{Introduction}

Quartz crystal microbalance (QCM) is known independently as a surface sensitive analytical device capable of on-line monitoring of interfacial reactions and thin film depositions $[1,2]$. QCM measures changes in acoustic thickness or mechanical resonance properties of a thin film deposited on a metal electrode (e.g. gold, silver, and copper, etc.) $[3,4]$. As a surface analytical tool, combined QCM data collection and analysis allow one to take the advantage of the strengths, to test the validity of the assumptions and to gain a better understanding of a specific interfacial reaction. QCM has been widely used for biological analysis, clinical diagnosis, and environmental monitoring [5,6]. The liquid cell configuration of this device makes it suitable for realtime study of bioaffinity reactions in relevant solution conditions of temperature, flow rate, $\mathrm{pH}$ and ionic strength. In the recent years, the applications of QCM for biological analyses have been reported increasingly, including immunoassay of BSA [7], enterotoxin detection [8], enzymatic analysis for hydrogen peroxide quantification [9], and blood plasma coagulation determination [10].

PG is a well-known synthetic antioxidant on the US Food and Drug Administration list (Figure 1). It is a derivative of gallic acid which is one of the effective ingredients of commonly used traditional Chinese medicine (TCM) Paeoniae Radix. Besides its antioxidant activity, PG has many pharmacological effects such as free radical elimination [11], inhibiting platelet aggregation [12,13], anti-inflammatory effects [14], and anti-tumor activities [15]. It is widely used to nourish blood, activate circulation, alleviate pain, regulate menstruation, treat liver disease and cancer. But until now, the researches on PG have been focused mainly on its curative effects at the holistic level. There are no detailed studies on the mechanisms and dynamics of action at the molecular level, which limits the further clinical use of PG.

Interleukin-6 (IL-6) is a multifunctional protein that mediates both immune and inflammatory responses. Recombinant human IL-6 is a kind of polypeptide containing 184 amino acid residues whose molecular weight is estimated to be $20.9 \mathrm{kDa}$ [16]. IL-6 is secreted by many cell types, activated monocytes, macrophages, T cells, endothelial cells and bone marrow stroma cells [17]. IL-6 is a cytokine with many functions, which induces the transformation of activated B cells into immunoglobulin-secreting cells and the productionof acute phase proteins by hepatocytes [18]. IL-6 acts on target cells via a receptor consisting of two transmembrane glycoproteins, gp80 and gp130. The binding of IL-6 to its specific receptor (gp80, IL-6R) triggers the dimerization of the signal-transducing receptor subunit gp130 [19].<smiles>CCCOC(=O)c1cc(O)c(O)c(O)c1</smiles>

Figure 1: Chemical structure of propyl gallate, Mr 213.

${ }^{*}$ Corresponding author: Jinghua Yu, Key Laboratory of Chemical Sensing \& Analysis in Universities of Shandong, School of Chemistry and Chemical Engineering, University of Jinan, Jinan 250022, P. R. China, E-mail: ujn.yujh@gmail.com

Received April 25, 2015; Accepted May 14, 2015; Published May 16, 2015

Citation: Liu S, Huang J, Chen Q, Yu J (2015) Use of a Quartz Crystal Microbalance for Studying the Effects of Propyl Gallate on the Interactions of Interleukin-6 and Its Soluble Receptor. Med chem 5: 192-196. doi:10.4172/2161-0444.1000263

Copyright: @ 2015 Liu S, et al. This is an open-access article distributed under the terms of the Creative Commons Attribution License, which permits unrestricted use, distribution, and reproduction in any medium, provided the original author and source are credited. 
The soluble form of the IL-6R (sIL-6R) when linked to IL-6 triggers the dimerization of gp130 and in contrast to several other soluble cytokine receptors, which inhibit ligand function, In fact, the IL-6/sIL$6 \mathrm{R}$ complex rather than IL- 6 is believed to be the active form in vivo [20]. The soluble IL-6/IL-6R complex can activate signal transduction and biological responses in cells that possess gp130 and do not express gp80 IL-6R [21,22]. IL-6 produced at sites of inflammation or in the peripheral blood in response to stimuli released by inflammatory sites (IL-1 or TNFa) binds the serum sIL-6R and is transferred in the blood as an IL-6/sIL-6R complex [23]. This binding protects IL-6 from protease degradation and stabilizes its bioactivity. The routine method to study on the interaction between IL-6 and sIL-6R is enzyme-linked immunodeficient assay (ELISA), which is complicated, time-cost, only detects the equilibrium binding values and cannot monitor the process of the binding in real time.

In the present paper, a QCM was employed to monitor the interaction between IL- 6 and sIL-6R. Moreover, to evaluate the affinity of the binding, the interactions between sIL-6R and IL-6 with series of concentrations were detected and the kinetic analyses between IL-6 and sIL-6R were carried out using FAST fit program. Furthermore, the effects of PG on IL-6/sIL-6R binding based on the interaction analysis are observed to find out the molecular mechanism of propyl gallate.

\section{Experimental}

\section{Materials}

$\mathrm{N}$-hydroxysuccinimide(NHS),1-ethyl-3-(3-dimethylaminopropyl) carbodiimide (EDC), ethanolamine and PG were purchased from Sigma-Aldrich (St Louis, MO, USA). Recombinant human Interleukin-6 (IL-6) and recombinant human Interleukin-6 soluble receptor (sIL-6R) were purchased from PeproTech EC (London, UK). AT-cut $9 \mathrm{MHz}$ QCM quartz crystal substrates were purchased from SEIKO EG\&G. Phosphate-buffered saline Tween-20 (PBST, pH7.4) was composed of $0.01 \mathrm{M} \mathrm{Na}_{2} \mathrm{HPO}_{4} / \mathrm{NaH}_{2} \mathrm{PO}_{4}, 0.138 \mathrm{M} \mathrm{NaCl}, 0.0027 \mathrm{M}$ $\mathrm{KCl}$ and $0.05 \%$ Tween-20. All solutions were made by using deionized water. All reagents were of analytical grade and were used without further purification.

\section{Apparatus}

The QCM system employed in this study consists of an AT-cut $9 \mathrm{MHz}$ quartz crystal substrate. Five $\mathrm{V}$ dc was applied to the circuit to drive the crystal substrate and the frequency was monitored with a frequency counter (Model QCA917, SEIKO EG $\triangleleft G$ ) connected to a computer. The quartz crystal was employed as a working electrode. The application of a small change in mass $\Delta m$ on the surface of the crystal results in a proportional shift in resonant frequency $\Delta F$. This relationship was firstly described by Sauerbrey [24] with the equation (1):

$$
\Delta F=-2 F_{0}{ }^{2} \Delta \mathrm{m} / \mathrm{A}\left(\rho_{q} \mu_{q}\right)^{1 / 2}
$$

Where $F_{0}$ is the fundamental oscillation frequency of the dry crystal, $\rho_{q}$ is the density of quartz $\left(2.65 \mathrm{~g} \mathrm{~cm}^{-3}\right)$ and $\mu_{q}$ is the shear modulus $\left(2.95 \times 10^{11}\right.$ dyne $\left.\mathrm{cm}^{-2}\right)$, A is the electrode area $\left(0.20 \mathrm{~cm}^{2}\right)$. For the $9 \mathrm{MHz}$ quartz crystals used in this work, equation (1) predicts that a frequency change of $1 \mathrm{~Hz}$ corresponds to a mass increase of $0.89 \mathrm{ng} \mathrm{mm}^{-2}$. It is a kind of transducer capable of sub-nanogram mass measurement in vacuo and gases [25]. They have been shown to be capable of the measurement of changes in solution properties [26,27].

\section{Procedures}

Immobilization of sIL-6R on the surface of the QCM gold substrates: The gold substrate surface was cleaned by placing a drop of Piranha solution $\left(3: 1 \mathrm{H}_{2} \mathrm{SO}_{4} / 30 \% \mathrm{H}_{2} \mathrm{O}_{2}\right)$ [28]. CAUTION: Piranha solution should be handled with extreme care and only small volumes should be prepared at any one time. Piranha solution was spread on the gold surface for $3 \mathrm{~min}$. Then, the substrate was rinsed thoroughly with deionized water. This process was repeated three more times.

Furthermore, the gold substrate was cleaned by using acetone for $30 \mathrm{~min}$ and incubated in anhydrous methanol for $30 \mathrm{~min}$. By the addition of $20 \mu$ of $\alpha$-TA solution on the surface of the substrate for $50 \mathrm{~min}$. Disulfides are known as a kind of molecules to form a stable self-assembled monolayer (SAM) on a gold surface owing to the strong $\mathrm{S}-\mathrm{Au}$ covalent bond [29].Then a SAM of $\alpha$-TA was formed on the gold substrate. After the treated substrate was rinsed with deionized water for 3 times, $\alpha$-TA treatment surface introducing $\mathrm{COOH}$ groups made possible to immobilize avidin through the formation of amide bonds. EDC/NHS mixture was dropped to the SAM of $\alpha$-TA on the surface of the gold substrate. The function of the EDC/NHS is to activate and promote the formation of the covalent linkages by forming the $\mathrm{N}$-hydroxysucciniimide ester (Scheme. 1).

Then different concentrations of sIL-6R $(10 \mu \mathrm{l})$ were added onto the $a$-TA-bound surface of the gold substrate for $50 \mathrm{~min}$ at room temperature. The carboxy1 on the SAM of $\alpha$-TA bound NHS under the action of EDC and created active ester intermediate which bound the primary amines of the protein to make amide bond. Thus, sIL$6 \mathrm{R}$ was covalently immobilized on the gold substrate. The optimum concentration of sIL-6R is discussed later. Then the substrate was rinsed thoroughly with deionized water (Scheme 1).

Detection of the binding between sIL-6R and Paeoniae IL-6: Unreacted NHS-esters were blocked by washing with $1 \mathrm{M}$ ethanolamine, $\mathrm{pH}$ 8.5. Ten $10 \mu \mathrm{l}$ of IL-6 solution was added onto the surface of the gold substrate modified by sIL-6R, equilibrating for $20 \mathrm{~min}$. The frequency response was recorded. The amount of the immobilized IL- 6 was determined by the equation (1). The progressed binding of IL- 6 on the sIL-6R-bound surface of the gold substrate is schematically depicted in Scheme 1.
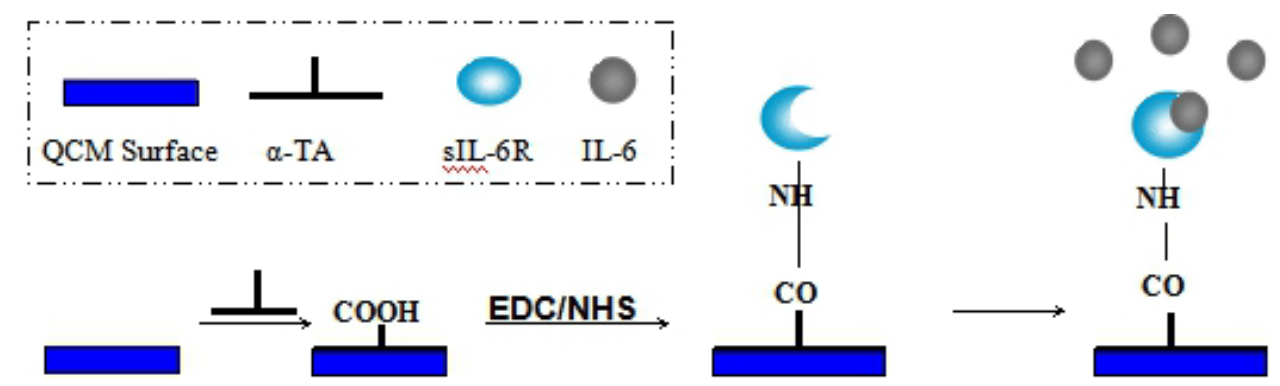

Scheme 1: The binding between ET-1 and Paeoniae Radix 801 on the surface of QCM. 
Kinetic Analysis of the Binding between IL-6 and sIL-6R: To analyze the kinetics of binding of IL-6 to sIL-6R, determination of kinetic and equilibrium constants was achieved by adding a wide range of ligate concentrations (in the ng- $\mu \mathrm{g} \mathrm{ml}^{-1}$ range) and allowing association to occur. Equilibrate sensor substrate for several minutes in $40 \mu \mathrm{l}$ of PBST, added $10 \mu \mathrm{l}$ of IL-6 solution into the cuvette, $10 \mathrm{~min}$ or so association was recorded. Remove the IL- 6 solution by washing the substrate 3 times with $50 \mu$ of PBST. Regenerate the bound IL- 6 by washing the substrate in $50 \mu \mathrm{l}$ of $10 \mathrm{mM} \mathrm{HCl}$, until the response signal returned to baseline. Wash sufficiently with PBST for re-use. Each binding cycle with different concentrations of IL-6 was analyzed as described above. Data obtained was analyzed using FAST fit program and $\mathrm{K}_{\mathrm{D}}$ was calculated.

Effects of Propyl Gallate on the Binding between IL-6 and sIL-6R: After three washes with PBST, $10 \mu \mathrm{l}$ of IL-6 $\left(0.01 \mathrm{mg} \mathrm{ml}^{-1}\right)$, preincubated for $15 \mathrm{~min}$ with propyl gallate concentration of 500 $\mathrm{nM}$, was added into the sIL-6R modified substrate. Then, detecte the binding between IL-6 and sIL-6R as described above.

\section{Results and Discussion}

\section{sIL-6R Immobilization on the Gold Substrates of QCM}

Figure 2 shows the response of a QCM immobilized by sIL-6R and IL-6 binding on the surface of the gold substrate. Response (1) is at the equilibration and a stabilized baseline after the addition of PBS. Response (2) corresponds to the addition of sIL-6R. Response (2) to (3) is buffer washing to remove unreacted sIL-6R. Response (4) is the blocking of non-coupled activated NHS-esters with ethanolamine. Response (5) is buffer washing to remove ethanolamine. Response (6) is the addition of IL-6. Response (7) is buffer washing to remove unreacted IL-6 (8) corresponds to $\Delta F$.

Response (1) is at the equilibration and a stabilized baseline after the addition of PBS. Response (2) to (3) is buffer washing to remove unreacted sIL-6R. Response (4) is the blocking of non-coupled activated NHS-esters with ethanolamine. Response (5) is buffer washing to remove ethanolamine. Response (7) is buffer washing to remove unreacted IL-6 (8) corresponds to $\Delta F$.

The mass of the gold substrate increased with the addition of a-TA on the surface of the gold substrate. The resonance frequency of QCM was simultaneously dropped. The gold substrate was rinsed with deionized water in order to remove the adsorbed species. The sIL-6R addition led a shift of response frequency to decreasing direction (2)(4), showing that sIL-6R was immobilized on the surface of substrate. The adsorbed values of sIL-6R on the surface of substrate were obtained as $277.858 \mathrm{ng} \mathrm{cm}^{-2}$ by the equation (1).

\section{Detection of the Binding between IL-6 and sIL-6R by QCM}

The subsequent addition of IL- 6 gave a typical irreversible response (6) and led a shift of resonance frequency to decreasing direction (6)-(8), indicating a time-dependent accumulation of the IL- 6 molecules on the surface. The adsorbed values of IL- 6 on the substrate were calculated as $130.919 \mathrm{ng} \mathrm{cm}^{-2}$. The results mentioned above are concluded that IL-6 binds sIL-6R.

The specific binding between IL- 6 and sIL-6R was evaluated by a blank control experiment without anchoring sIL-6R on the substrate surface. No detectable binding signal was obtained, showing that IL-6 binds sIL-6R specifically on the substrate surface.

\section{Kinetic analysis of the binding between IL-6 and sIL-6R}

Figure 3 shows the association curve of IL- 6 with different concentrations binding to immobilized sIL-6R. As IL-6 concentration increased, the initial binding velocity increased and the time taken to approach equilibrium decreased.

Data is analyzed using the FAST fit program, specifically designed for biomolecular interaction analysis using the IAsys optical biosensor. It performs the detailed mathematical analysis of the association and dissociation curves obtained in QCM. By specifying IL-6 concentration and the region to analyze and fitting the experimental data for each IL- 6 concentration to corresponding equations, the association curves showed good fitting to double phase association.

Each correctly-fitted association curve provides a value for $\mathrm{k}_{\text {on }}$ the apparent association rate constant, which was used to determine $\mathrm{k}_{\text {ass }}$ (association rate constant) and $\mathrm{k}_{\text {diss }}$ (dissociation rate constant), as described by:

$$
\mathrm{k}_{\mathrm{on}}=\mathrm{k}_{\text {ass }}[\mathrm{L}]+\mathrm{k}_{\text {diss }}
$$

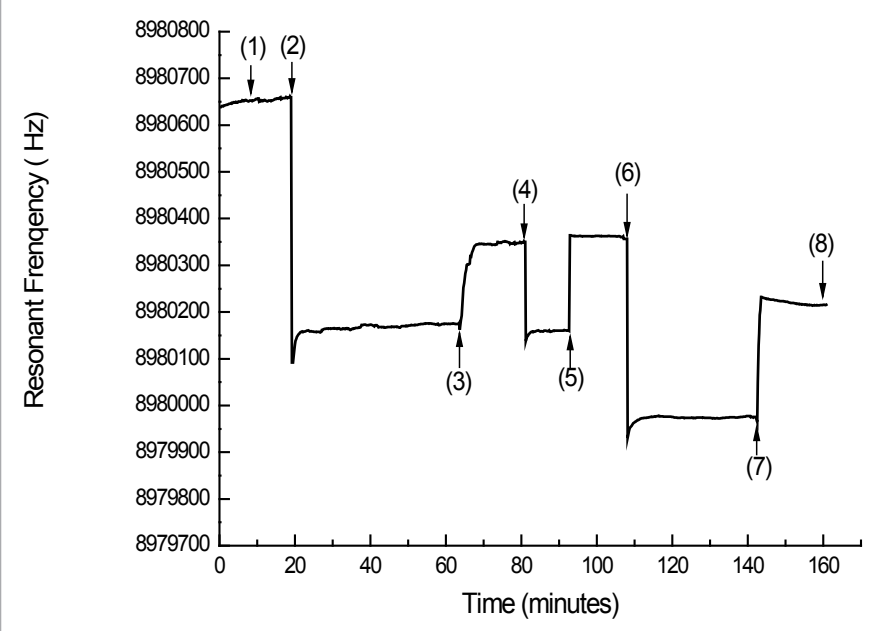

Figure 2: QCM sensorgram by immobilization of SIL-6R (2) - (4) and adsorption of IL-6 (6) - (8). Response (1) is at the equilibration and a stabilized baseline after the addition of PBS. Response (2) to (3) is buffer washing to remove unreacted sIL-6R. Response (4) is the blocking of noncoupled activated NHS-esters with ethanolamine. Response (5) is buffer washing to remove ethanolamine. Response (7) is buffer washing to remove unreacted IL-6 (8) corresponds to $\Delta F$.

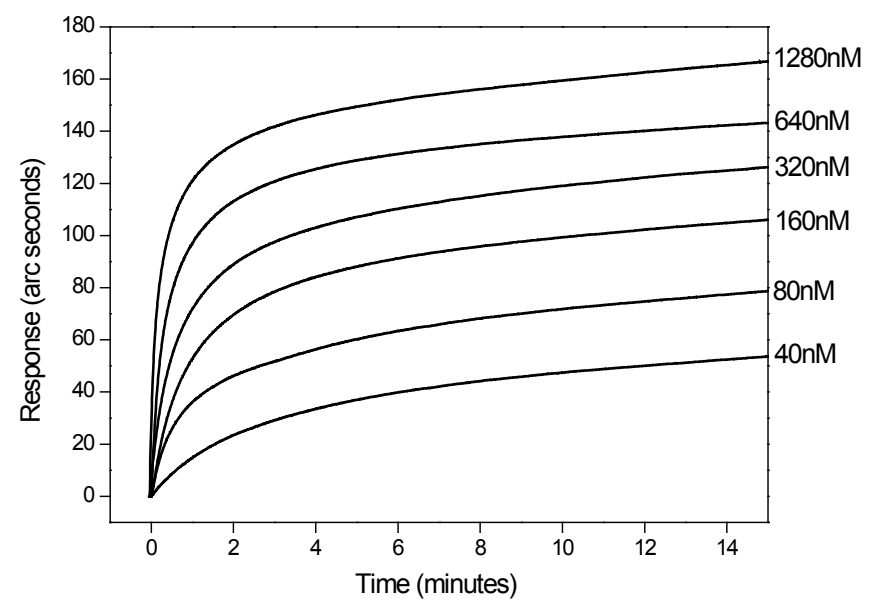

Figure 3: Association curve of IL-6 binding to immobilized sIL-6R. The concentrations of IL- 6 are $40 \mathrm{nM}, 80 \mathrm{nM}, 160 \mathrm{nM}, 320 \mathrm{nM}, 640 \mathrm{nM}, 1280 \mathrm{nM}$. 
these $\mathrm{k}_{\mathrm{on}}$ values are obtained for each concentration of ligate, IL-6. The certain option in FAST fit is used to produce a plot of $\mathrm{k}_{\text {on }}$ versus IL-6 concentration (Figure 4).

The gradient of the line in Figure 4 is used to calculate $\mathrm{k}_{\text {ass }}, \mathrm{k}_{\text {diss }}$ is obtained from the intercept of Figure 3. Thus $\mathrm{k}_{\text {ass }}=3.41 \times 10^{4} \mathrm{M}^{-1} \mathrm{~s}^{-1}$, $\mathrm{k}_{\mathrm{diss}}=1.80 \times 10^{-2} \mathrm{~s}^{-1} . \mathrm{K}_{\mathrm{D}}$ may be determined from the relationship:

$$
\mathrm{K}_{\mathrm{D}}=\frac{\mathrm{K}_{\text {diss }}}{\mathrm{K}_{\text {ass }}} \text {, thus } \mathrm{K}_{\mathrm{D}}=5.28 \times 10^{-7} \mathrm{M}, \mathrm{K}_{\mathrm{A}}=1 / \mathrm{K}_{\mathrm{D}}=1.89 \times 10^{6} \mathrm{M}^{-1} \text {, which }
$$

indicated that sIL-6R bind IL- 6 with high affinity. Compared to the $\mathrm{K}_{\mathrm{D}}$ of IL-6/sIL-6R binding, reported as $\sim 10^{-9} \mathrm{M}$ [27], it can be inferred that the binding ability of sIL-6R to IL- 6 is lower than sIL-6R. The results indicate that sIL-6R binding ability to IL-6 is decreased, which is probably attributable to the denaturation that occurred when sIL$6 \mathrm{R}$ was produced. Furthermore, the developed approach showed very desirable reproducibility. The relative standard deviations (RSDs) of $\mathrm{K}_{\mathrm{on}}\left(\mathrm{s}^{-1}\right)$ are $2.84 \%, 3.17 \%, 2.72 \%$, respectively, in three repetitive assays of $80 \mathrm{nM}, 320 \mathrm{nM}, 1280 \mathrm{nM}$ of TNF- $\alpha$. These data manifested that the developed approach held great potential with excellent reproducibility.

\section{Effects of Propyl Gallate on the Binding between IL-6 and sIL-6R}

Figure 5 showed the experimental results of the binding reactions of IL-6, with as well as without PG, on the immobilized sIL-6R surface. As PG involved, the initial velocity of IL-6/sIL-6R binding increased and the time taken to approach equilibrium decreased, which indicated that PG was able to enhance the binding of IL- 6 to sIL-6R.

\section{Conclusions}

In the present study, based on the interaction analyses between IL-6 and sIL-6R, the effects of PG on IL-6/sIL-6R binding were studied by using QCM. The results showed that IL-6 can bind sIL-6R specifically in a dose-dependent manner. Data achieved was analyzed using the FAST fit program, $\mathrm{K}_{\mathrm{D}}$ value is $5.28 \times 10^{-7} \mathrm{M}$, indicated that sIL-6R binds IL-6 with high affinity. Thus sIL-6R may protect cell and tissue form the pathologic injury of IL- 6 by competing the cell-surface receptor and neutralizing IL- 6 bioactivity, which agree with previous reports by others that the administration of sIL-6R can prevent the adverse pathologic sequelae of exaggerated IL production [30].

Moreover, the acquisition of kinetic analyses will be of benefit

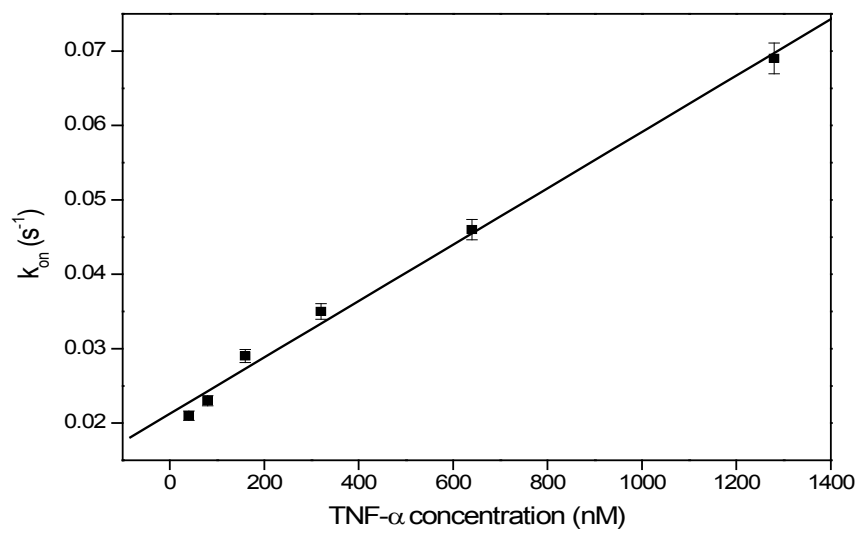

Figure 4: Plot of $\mathrm{k}_{\text {on }}$ against IL-6 concentration derived from association analysis of data from Fig. 5. $\mathrm{k}_{\text {ass }}=3.41 \times 10^{4} \mathrm{M}^{-1} \mathrm{~s}^{-1}, \mathrm{k}_{\text {diss }}=1.80 \times 10^{-2} \mathrm{~s}^{-1}$. Error bars are standard deviations across three repetitive experiments.

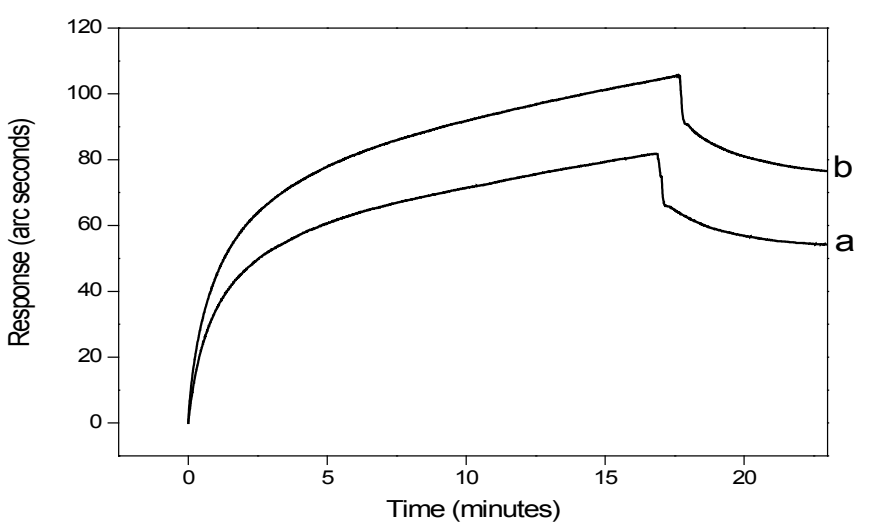

Figure 5: (a) The experimental results of the binding reactions of IL-6 without PG. (b) The experimental results of the binding reactions of IL-6 with PG.

for obtaining a better insight on the study on IL-6/sIL-6R system and further studies based on it. In this work, the effects of propyl gallate on interaction between IL-6 and sIL-6R were observed. The experimental results showed that PG enhanced IL-6/sIL-6R binding. Though the mechanism of PG is not clear and the roles of IL- 6 and sIL-6R are debated, it can be concluded that the binding between IL- 6 and sIL-6R is another target which PG can act on in vivo, that is, $\mathrm{PG}$ can enhance the cellular protection of sIL-6R through the increase neutralization to IL-6, which is in agreement with the experimental and clinical results reported [31].

A QCM was employed to explore the mechanisms of PG for the first time. This finding offers an effective way to study the biomolecular interaction as well as a new clue to the study of the mechanisms of PG based on cytokine/receptor binding. Though various methods are useful for studying the interaction process between cytokines and receptors, many of these methods require labeling of one of the two biomolecules with a fluorescent or radioactive tag and a long time to equilibrium. QCM appeared to be a high sensitive instrument for monitoring and quantifying the binding of the cytokine to the corresponding receptors, which is fundamental for obtaining a better insight on the molecular interactions between the cytokine and receptor involved in varieties of physiological and pathophysiologic processes. Furthermore there are other advantages of this new method, such as that the amount of both ligand and ligate needed to obtain informative results is very low, the time required to perform an assay is very short and all the procedures are label free. It has tremendous application prospect in the studies on the molecular mechanisms of TCM related to the cytokine/receptor interaction. Further studies in this area are in progress.

\section{Acknowledgements}

This work was financially supported by the National Natural Science Foundation of China (21475052, 21175058, 21277058) and the Technology Development Plan of Shandong Province, China (2014GGX103012).

\section{References}

1. Huang H, Ding LL, Ren HQ, Geng JJ, Xu K, et al. (2015) Preconditioning of Model Biocarriers by Soluble Pollutants: A QCM-D Study. ACS Appl Mater Interfaces 7: 7222-7230.

2. Durupthy O, Jeurgens LP, Bill J (2011) Biomimetic formation of titania thin films: effect of amino acids on the deposition process. ACS Appl Mater Interfaces 3: 1624-1632.

3. Dilushan RJ, Ronan JC, Paula EC (2013) In Situ and Real Time Characterization of Spontaneous Grafting of Aryldiazonium Salts at Carbon Surfaces. Chem Mater 25: 1144-1152.

4. Gou YZ, Stacy S, Geng J, Lenny V, David MH (2012) Controlled Alternate 
Citation: Liu S, Huang J, Chen Q, Yu J (2015) Use of a Quartz Crystal Microbalance for Studying the Effects of Propyl Gallate on the Interactions of Interleukin-6 and Its Soluble Receptor. Med chem 5: 192-196. doi:10.4172/2161-0444.1000263

Layer-by-Layer Assembly of Lectins and Glycopolymers Using QCM-D. ACS Macro Lett 1: 180-183.

5. Graf G, Kocherbitov V (2013) Determination of sorption isotherm and rheological properties of lysozyme using a high-resolution humidity scanning QCM-D technique. J Phys Chem B 117: 10017-10026.

6. Fuchiwaki Y, Yabe Y, Adachi Y, Tanaka M, Abe K, et al. (2014) Inkje monitoring technique with quartz crystal microbalance (QCM) sensor for highly reproducible antibody immobilization. Sens Actuators A 219: 1-5.

7. Wu D, Han D, Steckl AJ (2010) Immunoassay on free-standing electrospun membranes. ACS Appl Mater Interfaces 2: 252-258.

8. Tang DP, Tang J, Su BL, Chen GN (2010) Ultrasensitive Electrochemical Immunoassay of Staphylococcal Enterotoxin B in Food Using EnzymeNanosilica-Doped Carbon Nanotubes for Signal Amplification. J Agric Food Chem 58: 10824-10830.

9. Bahrini C, Herbinet O, Glaude PA, Schoemaecker C, Fittschen C, et al. (2012) Quantification of hydrogen peroxide during the low-temperature oxidation of alkanes. J Am Chem Soc 134: 11944-11947.

10. Doudrick K, Corson N, Oberdorster G, Eder AC, Herckes P, et al. (2013) Extraction and quantification of carbon nanotubes in biological matrices with application to rat lung tissue. ACS Nano 7: 8849-8856.

11. Hirayama M, Toda R, Ozaki T, Hasegawa J, Nakamura T, et al. (2011) Concentration Dependence of 5-Aminosalicylic Acid Pharmacological Actions in Intestinal Mucosa after Oral Administration of a pH-Dependent Formulation. Mol Pharmaceutics 8: 1083-1089.

12. Chou TC, Shih CY, Chen YT (2011) Inhibitory effect of a-lipoic acid on platelet aggregation is mediated by PPARs. J Agric Food Chem 59: 3050-3059.

13. Lee DS, Kim TH, Jung YS (2014) Inhibitory effect of allyl isothiocyanate on platelet aggregation. J Agric Food Chem 62: 7131-7139.

14. Uzma S, Bashir A, Mobasher A, Khalid H, Nadeem IB (2015) Anti-nociceptive, anti-inflmmatory and anti-pyretic activities of latex and leaves methanol extract of Euphorbia helioscopia. Asian Pac J Trop Dis 5: 322-328.

15. Gaowa A, Horibe T, Kohno M, Tabata Y2, Harada H3, et al. (2015) Enhancement of anti-tumor activity of hybrid peptide in conjugation with carboxymethyl dextran via disulfide linkers. Eur J Pharm Biopharm 92: 228-236.

16. Nielsen AR, Plomgaard P, Krabbe KS, Johansen JS, Pedersen BK (2011) IL6 , but not TNF-Ît, increases plasma YKL-40 in human subjects. Cytokine 55: 152-155.

17. Jingchao H, Rong S, Zhongchen S, Lan C (2011) Human amelogenin upregulates osteogenic gene expression in human bone marrow stroma cells. Biochem Biophys Res Commun 408: 437-441.

18. Ashida T, Kikuchi T1 (2015) Overview of binding free energy calculation techniques for elucidation of biological processes and for drug discovery. Med Chem 11: 248-253.
19. Li H, Xiao H, Lin L, Jou D, Kumari V, et al. (2014) Drug Design Targeting Protein-Protein Interactions (PPIs) Using Multiple Ligand Simultaneous Docking (MLSD) and Drug Repositioning: Discovery of Raloxifene and Bazedoxifene as Novel Inhibitors of IL-6/GP130 Interface. J Med Chem 57: 632-641.

20. José RL, Luis RR, Pilar TE, Esther V, José H, et al. (2013) Alternative splicing and proteolytic rupture contribute to the generation of soluble IL-6 receptors (sIL-6R) in rheumatoid arthritis. Cytokine 61: 720-723.

21. Rantala A, Lajunen $T$, Juvonen $R$, Silvennoinen-Kassinen $S$, Peitso $A$, et al (2011) Association of IL-6 and IL-6R gene polymorphisms with susceptibility to respiratory tract infections in young Finnish men. Hum Immunol 72: 63-68.

22. Ireland SJ, Monson NL2, Davis LS3 (2015) Seeking balance: Potentiation and inhibition of multiple sclerosis autoimmune responses by IL- 6 and IL-10. Cytokine 73: 236-244.

23. Silva Almeida IL, Melo Coelho NM (2012) Direct Determination of Inorganic Mercury in Ethanol Fuel by Cold Vapor Atomic Absorption Spectrometry. Energy Fuels 26: 6003-6007.

24. Sauerbrey S, Majhi PK, Daniels J, Schnakenburg G, Brandle GM, et al. (2011) Synthesis, structure, and reactions of 1-tert-butyl-2-diphenylphosphinoimidazole. Inorg Chem 50: 793-799.

25. Qian Y, Wu M, Wang W, Chen B, Zheng H, et al. (2015) Determination of 14 nitrosamines at nanogram per liter levels in drinking water. Anal Chem 87: 1330-1336.

26. Latif U, Can S, Hayden O, Grillberger P, Dickert FL (2013) Sauerbrey and antiSauerbrey behavioral studies in QCM sensors-Detection of bioanalytes. Sens Actuators B 176: 825-830.

27. Feng S, Zhang YZ, Chen Q, Zuo C, Li RB, et al. (2014) General solution for high dynamic range three-dimensional shape measurement using the fringe projection technique. Opt Laser Eng 59: 56-71.

28. Gupta RK, Srinivasan MP, Dharmarajan R (2011) Synthesis of short chain thiol capped gold nanoparticles, their stabilization and immobilization on silicon surface. Colloid Surface A 390: 149-156.

29. Selvaraju K, Jothi M, Kumaradhas $P$ (2012) Exploring the charge density distribution and the electrical characteristics of Oligo phenylene ethylene molecular nanowire using quantum chemical and charge density analysis. Comput Theor chem 996: 1-10.

30. Luethi E, Nguyen KT, BÃ $1 / 4$ rzle M, Blum LC, Suzuki Y, et al. (2010) Identification of selective norbornane-type aspartate analogue inhibitors of the glutamate transporter 1 (GLT-1) from the chemical universe generated database (GDB) J Med Chem 53: 7236-7250.

31. Lin CM, Shyu KG, Wang BW, Chang H, Chem YH, et al. (2010) Chrysin Suppresses IL-6-Induced Angiogenesis via Down-regulation of JAK1/STAT3 and VEGF: An in Vitro and in Ovo Approach. J Agric Food Chem 58: 70827087. 